\title{
Berry fillers based on local plant raw materials
}

\author{
Svetlana Gabelko*, and Olesya Ionova \\ Novosibirsk State Technical University, 20, K. Marx Prospekt, 630073 Novosibirsk, Russia
}

\begin{abstract}
When developing a diet for citizens with diseases of the gastrointestinal tract or obesity, it is necessary to adhere to a therapeutic diet in which bakery products and dairy products with berry fillers can be wholesome ingredients. In order to increase the range of berry fillers based on local plant raw materials, the physicochemical quality indicators were studied and the organoleptic evaluation of fillers with various types of thickeners was given.
\end{abstract}

\section{Introduction}

In the course of previous studies [1], it was revealed that about $45 \%$ of the population of the Russian Federation have one or more diseases related to nutrition and $16.1 \%$ of them have diseases of the gastrointestinal tract, with $7.3 \%$ of them being allergic to food. The mortality caused by the progression of most of these diseases can be decreased by reducing various risk parameters including hypertension, obesity, tobacco and alcohol use, diabetes, unhealthy eating habits, physical inactivity, hyperlipidemia, salt restriction, and increased consumption of fruits and vegetables in the diet [2]. The growing number of overweight and obese people across all age groups has become a major public health concern along with the related noncommunicable diseases caused by obesity, which resulted in deaths of 4 million people worldwide. In 2016, more than 1.9 billion adults worldwide were classified as overweight, and more than $34 \%$ of this population were obese (World Health Organization, 2020). Obesity is a complex disease that is caused by many factors, such as behavioral, genetic, and environmental factors. This is caused by low energy consumption due to a sedentary lifestyle or a genetic predisposition.

To address these challenges, the United Nations Decade for Action on Nutrition (20162025) focuses on promoting sustainable and healthy diets and increasing their nutritional value in accordance with the Sustainable Development Goals [3]. The consumption of a healthy and sustainable diet opens up wide opportunities for reducing greenhouse gas emissions by food and processing enterprises and for improving health indicators of the population [4].

When drawing up diets for citizens, it is necessary to adhere to a therapeutic diet where bakery and dairy products with fruit and berry fillers can be wholesome ingredients.

In line with consumer demands, there is a growing interest in developing new foods with a more balanced composition, including a low sugar content that provide additional health benefits due to bioactive compounds [5]. Fruit and berry fillers made from natural raw materials combine factors that promote the activity of the enzyme systems of the

\footnotetext{
${ }^{*}$ Corresponding author: gabelko@,corp.nstu.ru
} 
intestinal microflora and enrich the diet with substances that increase the body's resistance to negative environmental factors.

Since the composition of berries and fruits is dominated by water, they do not have a high energy value. The main energy material in the composition of fruits and berries are easily digestible carbohydrates that predominate in the dry residue. Fruits and berries are of the greatest nutritional value as a source of biologically active substances, vitamins, macro and microelements, and dietary fiber [6].

Fruits and berries have a high antioxidant capacity [7]. Medical studies have revealed the medicinal properties of pigmented polyphenols, such as flavonoids, anthocyanins, tannins, and other phytochemicals, mainly localized in the peel and seeds [8].

Numerous epidemiological and clinical studies demonstrate the beneficial effect of natural polyphenol supplements on the cardiovascular system, and the slowing down of human biological aging [9]. It is known that the polyphenols contained in fruit and berry raw materials interfere with the activity of key digestive enzymes, which, in turn, reduce the digestion of carbohydrates and fats, thereby reducing energy consumption [10].

can be used to prevent a number of diseases, including cardiovascular diseases, cancer, diabetes, certain metabolic diseases, and microbial infections. These compounds improve human visual abilities and have a neuroprotective effect [11].

The products of heat processing of berries contain less polyphenols compared to fresh berries, but their total content remains relatively high [12]. Acylated and copigmented anthocyanidins have higher heat resistance and retain their structure at different $\mathrm{pH}$ levels [13].

Fruits and berries are considered as a source of dietary fiber and pectin substances entering the human body. Dietary fiber intake has beneficial health effects, including a reduction in the incidence of cardiovascular disease, diabetes, colon cancer, obesity, and some gastrointestinal disorders, and appears to improve immune function [14]. Pectin has the ability to cleanse the body of harmful substances, without disturbing the bacteriological balance of the body. Due to the ability of pectin substances not to be broken down under the action of stomach enzymes, to interact with various metal ions and radionuclides and remove them from the body, pectins are used as a preventive agent for intoxication [6].

Fruit and berry fillers are widely used in various branches of the food industry. Confectionery production uses fruit and berry fillers in the manufacture of cakes, pastries, bakery products. In dairy production, fillers are used as additives in yoghurts, cheeses, and ice cream. In retail trade, fruit and berry fillers come as an independent product in the form of low-calorie jams, jams, various gelled products.

Currently, the market for fillers is dominated by products with a high sugar content and artificial food additives. Therefore, the task of developing fruit and berry fillers from local plant raw materials with improved nutritional characteristics while maintaining functional and technological properties is urgent.

The purpose of this work was to develop recipes and technologies for fillers from local plant raw materials, taking into account consumer preferences, and to evaluate the organoleptic and physico-chemical quality indicators of fillers made using various thickeners.

\section{Objects and methods of research}

\subsection{Research objects}

The objects of the study were fruit and berry fillers made from raw materials of the Siberian region. These fillers are a boiled viscous mass of mashed berries and fruits, or a mass with inclusions of whole berries and pieces of fruit. Frozen local vegetable raw materials were 
used as the main component (50\%) of fruit and berry fillers, which significantly reduced their caloric content and increased the amount of fiber and biologically active substances. In addition to the fruit and berry part and sugar (30 ...33\%), the fillers included thickeners, citric acid, preservatives (potassium sorbate), and flavors identical to natural ones.

Pectin is the main food thickener for fruit and berry fillings which combines perfectly with other hydrocolloids. When developing filler formulations, thermoreversible lowesterified pectins $\mathrm{NH}, \mathrm{NH}+$ and modified corn starch having the most appropriate properties were used as thickeners. Thermoreversible pectins are able to melt repeatedly without reducing the quality, so they are used to produce glazes, sweet sauces and fillings based on fruit pulp, including those with a high moisture content. Low-esterified pectins are less sensitive to $\mathrm{pH}$ than standard pectin gels, with conditions for gel formation for them being $\mathrm{pH}$ 2.5...6.5. Gelation of low-esterified pectins does not require sugar, but the addition of $10 \ldots 20 \%$ sucrose improves the gel structure [15]. Amylopectin corn starch has a lower temperature of the beginning of gelatinization than the native one. The paste from it is transparent, resistant to excessive acidity and mechanical influences. Amylopectin swollen starch is distributed evenly over the volume forming transparent stable systems. Therefore, swollen ("cold soluble") starches are used as thickeners for dry fast food products, various concentrates, soups, sauces, candy fillings, pies, confectionery creams, etc. [16].

\subsection{The sociological method of research}

To study the preferences of the target audience regarding the characteristics of fruit and berry fillers, a social survey was conducted among 130 full-time students of secondary special and higher educational institutions aged 15 to 23 years.

\subsection{Organoleptic and physico-chemical methods of research}

Organoleptic and physico-chemical indicators of the quality of fillers were determined in compliance with GOST 54682-2011 [17] by the following methods: the mass fraction of titrated acids was found by titration in the presence of the timolphthalein indicator, the mass fraction of soluble solids was found by the refractometric method (the IRF-454 B2M refractometer, Russia), the $\mathrm{pH}$ was found by the $\mathrm{pH}$ meter $150 \mathrm{MI}$ with a combined electrode ESK-10603/7, with the range of measurements of the activity of hydrogen ions being from 0 to $14 \mathrm{pH}$ units. and with the limit of the permissible absolute measurement error of $\pm 0.05 \mathrm{pH}$ units (Russia).

\section{Analysis of the results obtained}

During the social survey, it was found that $51 \%$ of respondents when choosing products with a filling paid attention to their caloric content (Fig. 1), while for $38 \%$ of respondents, the amount of sugar in the product was also important (Fig. 2). The majority of respondents when using products preferred berry fillings from strawberries, cherries and raspberries (Fig. 3), while $71 \%$ of them preferred heterogeneous fillings (the presence of inclusions in the form of berries or fruits), and $29 \%$ preferred homogeneous fillings. 
- whatever $\leq 200$ calories $\quad 200-300$ calor

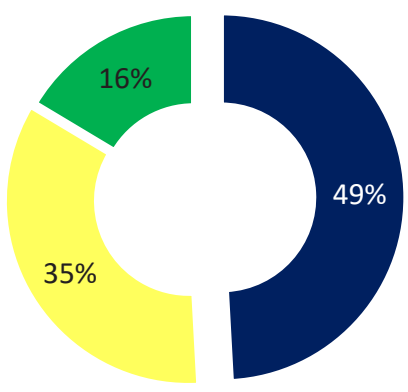

Fig. 1. Consumer choice of bakery products with fruit and berry fillings depending on their caloric content. $\leq 10 \mathrm{~g} . \quad 10-15 \mathrm{~g} . \quad \geq 15 \mathrm{~g} . \quad$ whatever

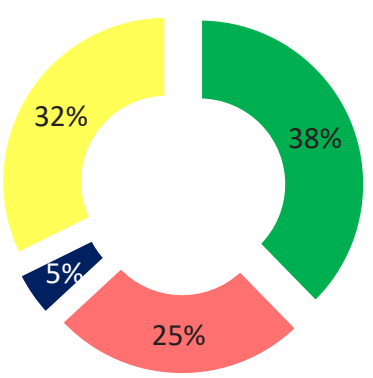

Fig. 2. Consumer choice of bakery products with fruit and berry fillings according to their sugar content.

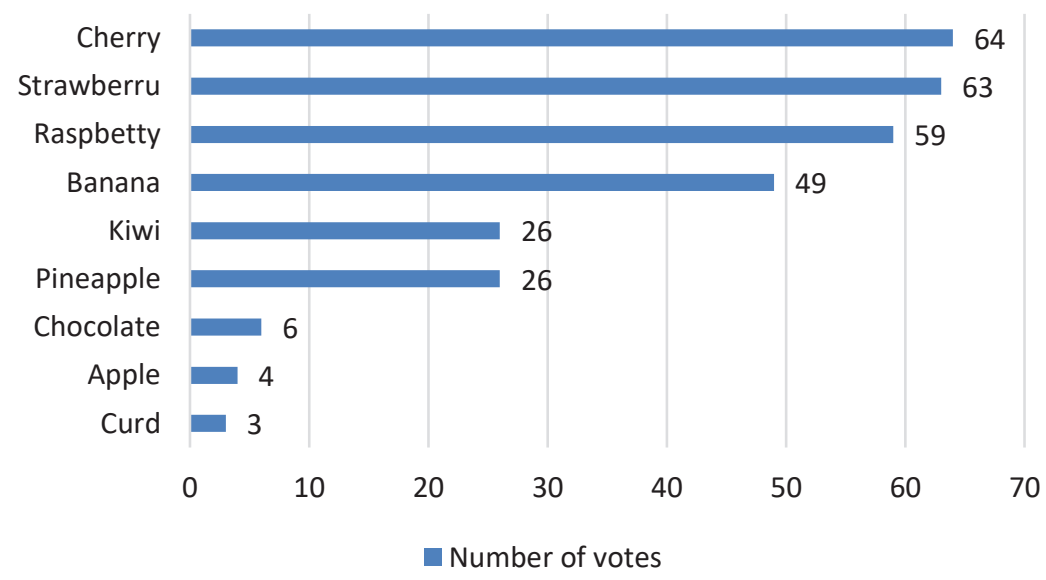

Fig. 3. Consumer preferences of fruit and berry fillings in the product recipe.

Based on the survey data and taking into account the range of fillers produced by manufacturers and used in food enterprises, recipes for berry fillers that meet the consumer 
preferences of the target audience were developed. The most popular fruit and berry fillers were fillers with reduced calories and low sugar content from strawberries, cherries, raspberries with pieces of fruit and berries. Table 1 shows an example of the recipe of the developed filling from cherries and strawberries cultivated in the Siberian region.

Table 1. Berry filling (Cherry (strawberry)

\begin{tabular}{|l|c|c|c|}
\hline \multirow{2}{*}{ Raw material name } & \multirow{2}{*}{$\begin{array}{c}\text { Mass fraction of } \\
\text { dry substances, } \\
\text { \% }\end{array}$} & life-size & $\begin{array}{l}\text { Consumption of raw materials g } \\
\text { substances }\end{array}$ \\
\cline { 4 - 4 } & & 500,0 & 75,0 \\
\hline Cherry, strawberry & 15,00 & 327,6 & 327,1 \\
\hline Sugar & 99,85 & 12,0 & 10,4 \\
\hline Pectin NH & 87,00 & 1,0 & 0,99 \\
\hline Potassium Sorbate & 99,00 & 1,0 & 0,98 \\
\hline Citric acid & 98,00 & 0,5 & 0,0 \\
\hline Flavor "Cherry" ("Strawberry") & 0,00 & 157,9 & 0,0 \\
\hline Water & 0,00 & 1000,0 & 414,5 \\
\hline Total & - & & \\
\hline
\end{tabular}

In order to preserve the integrity of the berries, it was decided to introduce $\mathrm{NH}+$ pectin and modified corn starch in the amount of $0.2 \%$ and $3 \%$, respectively. At this concentration of thickeners, the filling did not hold its shape well during baking $\left(\mathrm{t}=200^{\circ} \mathrm{C}\right)$. With an increase in the content of $\mathrm{NH}+$ pectin to $0.4 \%$, the filling began to keep its shape when baking products, did not spread and the berries retained their shape.

The technological phase of the production of fruit and berry filler is shown in Fig.4.

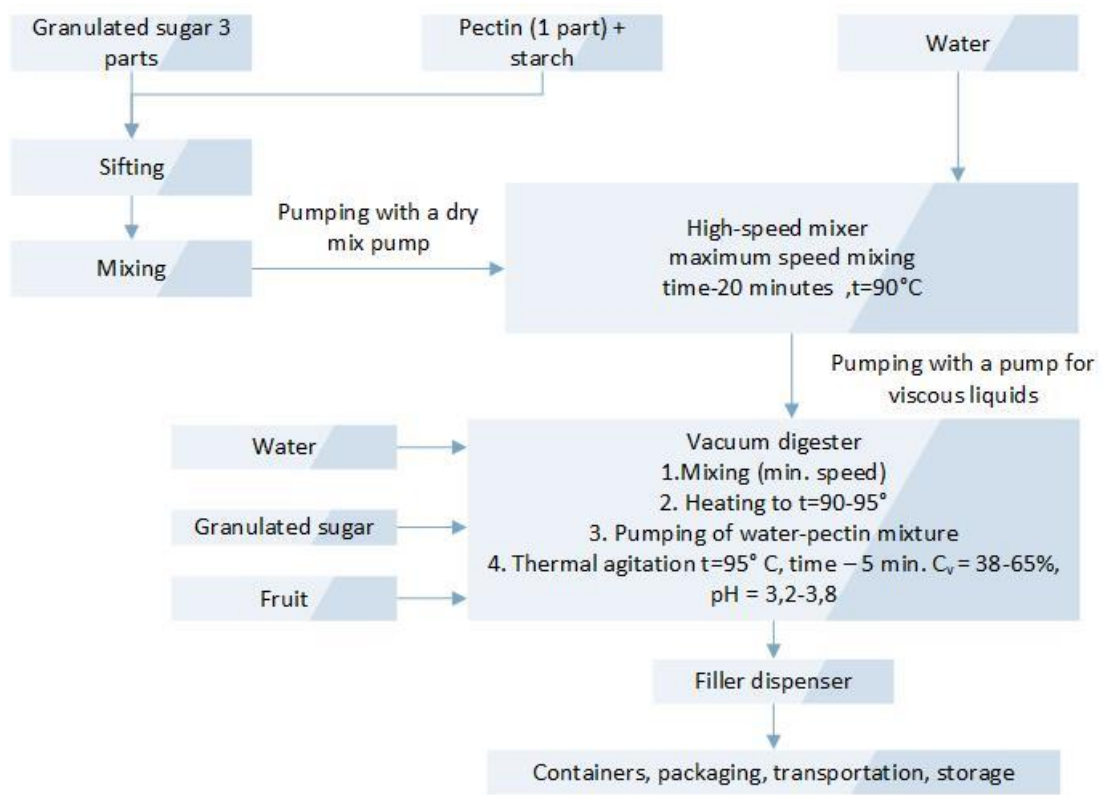

Fig. 4. Process chart of fruit and berry filler production.

According to organoleptic parameters, the fillers were characterized by a pronounced sweet and sour taste of strawberries and cherries; the color characteristic of berries that have undergone heat treatment; the consistency with a thickener pectin $\mathrm{NH}$ was fairly elastic and dense and with a glossy surface. When the thickener pectin $\mathrm{NH}+$ was used, the consistency was more jelly- like compared to a filler with a thickener pectin $\mathrm{NH}$, and had a 
glossy surface. However, when modified corn starch thickeners with pectin $\mathrm{NH}+$ were used, the consistency was most gelatinous and delicate with a less glossy surface than the other samples studied.

The mass fraction of dry substances in the studied samples of berry fillers was $32 \pm 0.5 \%$.

The results of determining the mass fraction of titrated acids in berry fillers with an error of $\pm 0.01 \%$ are shown in Fig. 5 .

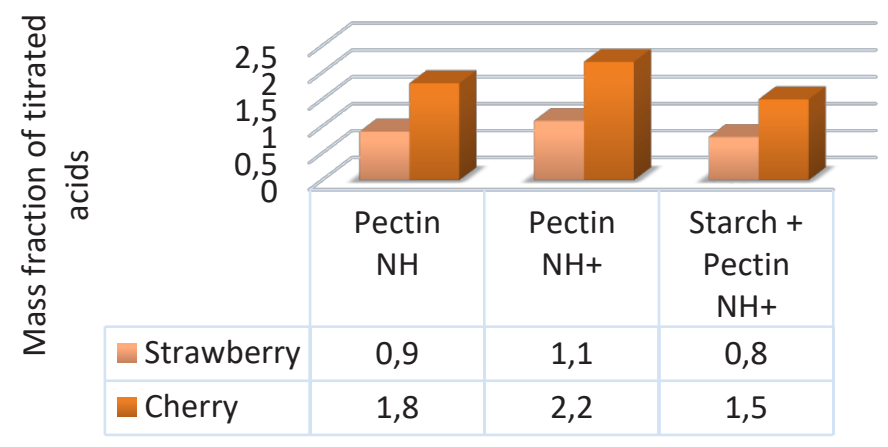

Fig. 5. The results of determining the mass fraction of titrated acids in the samples of berry fillers under study.

The results of $\mathrm{pH}$ determination with an error of \pm 0.02 are shown in Fig. 6 .

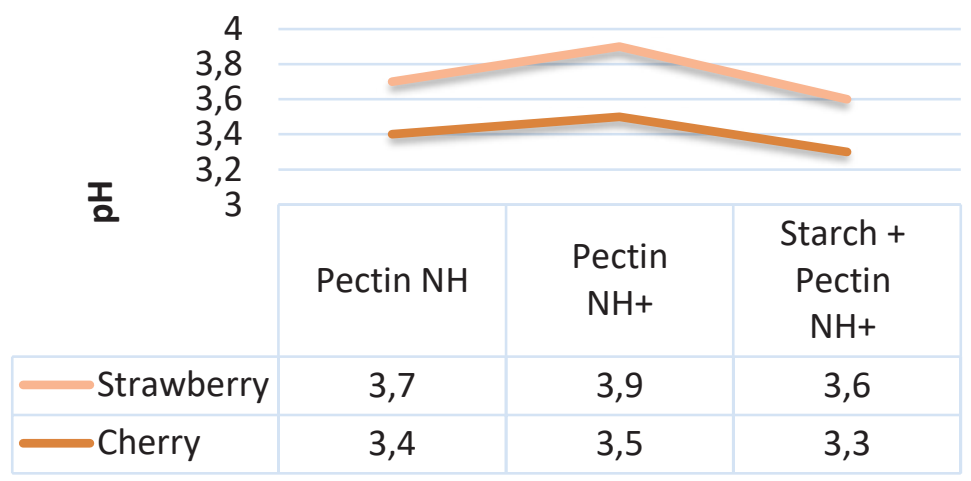

Fig. 6. The results of $\mathrm{pH}$ determination in the studied samples of berry fillers.

According to GOST R 54682-2011 [17] the values of the mass fraction of soluble solids should be in the range from $20.0 \%$ to $70 \%$, the mass fraction of titratable acids should be from $0.5 \%$ to $2.5 \%$, and $\mathrm{pH}$ should be above 4.2 .

Analyzing the data of physico-chemical studies of sample fillings, it can be concluded that the values of the quality indices are within the established norms.

\section{Conclusions}

The experiment showed that the fillers according to the recipes, including pectins $\mathrm{NH}$ and $\mathrm{NH}+$, have good organoleptic properties, within the established norms of physico-chemical quality indicators and a limited thermal stability. The low thickening capacity of pectin $\mathrm{NH}+$ during cooking makes it possible to keep the berries whole and get a beautiful, transparent gel. 
Based on the results of the study, it is possible to recommend the use of berry fillers with a thickener:

- pectin NH- for the preparation of confectionery products without subsequent heat treatment due to the firm and elastic consistency,

- pectin $\mathrm{NH}+$ - for the preparation of flour confectionery products with subsequent heat treatment,

- $\quad$ pectin $\mathrm{NH}+$ with modified corn starch - for the dairy industry in the preparation of yoghurts and desserts.

The introduction of the developed recipes and technology of berry fillers will allow a greater use of local wild and cultivated plant raw materials, thereby contributing to the conservation of biodiversity and the development of regional food markets. Maintaining a high level of biodiversity, in turn, provides a full human diet with a sufficient average level of consumption of food and biologically active substances.

Thus, the results obtained meet the current needs of consumers and are aimed at achieving the goals of sustainable development of the region.

\section{References}

1. The diet of the population. 2013: Statistical Yearbook (New York: IIC "Statistics of Russia", 2016)

2. T. Behl, Bungau, S., K. Kumar, G. Zengin, F. Khan, A. Kumar, R. Kaur, T. Venkatachalam, D. M. Tit, C. M. Vesa, G. Barsan, D.-E. Mosteanu, Biomedicine \& Pharmacotherapy Is, 130 (2020)

3. FAO and WHO. 2020. Sustainable Healthy Eating-Guidelines. Rome

4. IPCC. 2019. Climate Change and Land Resources: IPCC Special Report on climate change, desertification, land degradation, sustainable land use, food security, and greenhouse gas fluxes in terrestrial ecosystems, https://www.ipcc.ch/ report/srccl/

5. Raquel P. F. Guiné, Paula M. R. Correia, Cátia Reis and Sofia G. Florença, De Gruyter, 8 (2020)

6. I. E. Tsapalova, M. D. Gubina, O. V. Golub, V. M. Poznyakovsky, Examination of wild fruits and berries and herbaceous plants, Quality and safety (Novosibirsk: Siberian University Publishing House, 2005)

7. So Janpath, G. Barba-Espin, R. Muller, B. Jernigan, John. N. Hegelund, B. Madsen at al., Food Biosci. (2020)

8. D. Donno, M. Cavanna, G. L. Beccaro, G. M Mellano, D. T. Marinoni, A. K. Cerutti, G. Bounous, Journal of Applied Botany and Food Quality, 86 (2013)

9. S. Esposito, Al. Gialluisi, S. Costanzo, Nutrients, 13-5 (2021)

10. M. Singh, T. Thrimawithana, R. Shukla, B. Adhikari, ScienceDirect, 1-2 (2020)

11. J. Renaud, Maria-Grazia Martinoli, IJMS, 20-8 (2019)

12. B. Levaj, V. Dragović-Uzelac, K. Delonga, K. K. Ganić, M. Banović, D. B. Kovačević , Food Technology and Biotechnology, 48(4) (2010)

13. H. E. Khoo, A. Azlan, FOOD \& NUTRITION RESEARCH, 61 (2017)

14. A. M. Bernstrand, Didieri, D. Garssen, D. K. Wurster, research in the field of food and nutrition, 61-1 (2017)

15. A. P. Nechaeva, Food chemistry (St. Petersburg: GIORD, 2007)

16. E. K. Koptelova, N. D. Lukin, Yu. I. Tretyakov, Food industry, 4 (2012) 
17. GOST R 54682-2011. Semi-finished products. Fruit and vegetable fillers. General technical conditions. 